\title{
En busca de la autonomía tecnológica. La trayectoria de la Empresa Nuclear Argentina de Centrales Eléctricas S. A., 1980-1996
}

\section{In Search of Technological Independence. The Trajectory of the Empresa Nuclear Argentina de Centrales Eléctricas S. A., 1980-1996}

Milagros Rocio Rodríguez ${ }^{1, *}$ (iD 0000-0001-8879-5298

${ }^{1}$ Universidad de Buenos Aires, Buenos Aires, Argentina.

*Correspondencia: mily_89r@hotmail.com

Resumen. Con base en los lineamientos del concepto autonomía tecnológica, la Empresa Nuclear Argentina de Centrales Eléctricas S. A. (Enace S. A.), constituida en 1980 a partir del consorcio entre la Comisión Nacional de Energía Atómica y Siemens Kraftwerk Union, intentó conformar un Arquitecto Industrial encargado de la instalación de Centrales Nucleares, además de promover el entrenamiento de personal argentino, incrementar la participación de la industria local y adquirir el know how de la ingeniería y el diseño de los reactores. Sin embargo, a pesar de instrumentarse políticas de corte neoliberal, la empresa perdió su sentido original y fue disuelta en 1996. Este trabajo apunta a realizar una aproximación general a través del análisis de las fuentes disponibles, pues la producción historiográfica sobre el tema resulta prácticamente inexistente.

CÓMO CITAR: Rodríguez, M. R. (2020). En busca de la autonomía tecnológica. La trayectoria de la Empresa Nuclear Argentina de Centrales Eléctricas S. A., 1980-1996. América Latina en la Historia Económica, 28(1), 1-22. DOI: https://doi.org/10.18232/alhe.1097 
Palabras clave: Argentina; Estado; Empresas Públicas; Desarrollo Económico; Industria Nuclear.

Abstract. The Empresa Nuclear Argentina de Centrales Eléctricas S. A. (Enace S. A.) was a consortium created in 1980 between the Comisión Nacional de Energía Atómica and Siemens Kraftwerk Union. Following the guidelines of technological independence, the joint venture seeked to operate as an Industrial Architect aimed at the construction of Nuclear Power Plants, as well as promote the training of national personnel, increase the participation of local industry and acquire know-how on reactor engineering and design. However, as neoliberal policies began to be implemented, the company lost its original purpose and was finally dissolved in 1996. Given that historiography on the subject is scarce, this work aims to offer a general approach through the analysis of the available sources.

Key words: Argentina; State; Public Companies; Economic Development; Nuclear Industry.

JEL: N16; N56; N76; N96.

Recibido: 30 de julio de 2019

Aceptado: 26 de septiembre de 2020

Publicado: 23 de noviembre de 2020.

\section{INTRODUCCIÓN}

\section{Energía nuclear y desarrollo en la periferia}

En líneas generales, el crecimiento económico global ocurrido durante la posguerra se encuentra estrechamente ligado al incremento de la demanda energética, en tanto que suele identificarse una relación estable entre las fluctuaciones del PBI y el consumo energético per cápita. No obstante, no todas las formas de producir energía tienen el mismo impacto económico y, es en este sentido, que la generación nucleoeléctrica, nacida en la década de 1950, se ha transformado en un símbolo de "progreso" y "desarrollo" (Rubio-Varas y De la Torre 2017). Dado que el sector nuclear requiere de una estructura sólida de formación de recursos humanos, así como de facilidades abocadas a la elaboración y el tratamiento de elementos combustibles, la multiplicación de usinas estimuló el desarrollo paralelo de la industria nuclear y convencional (Frewer y Altvater, 1977). Por este motivo, si bien los Estados Nacionales han desempeñado un rol protagónico con la creación de Comisiones de Energía Atómica, la participación de las empresas nacionales y el desarrollo de los ámbitos de producción de Ciencia y Tecnología (CyT) han constituido aspectos cruciales para asegurar el desarrollo exitoso del sector. Allí donde los lazos entre las partes involucradas del "triángulo de hierro" (iron triangle) -el gobierno, la ciencia y los grupos de interésresultaron fluidos, así la expansión de la energía nuclear pudo alcanzar una maduración plena (Balogh, 1991; Rubio-Varas y De la Torre, 2014). Sin embargo, no se verificó la misma tendencia en los países de la periferia, donde la desconexión entre los tres vértices ha sido señalada como una de las causas del subdesarrollo y del atraso tecnológico. En este contexto, los programas nucleares, adoptados tempranamente en Brasil, México, España, India y Argentina, adquirieron un nuevo sentido en función de la necesidad de profundizar el proceso de industrialización por sustitución de importaciones. Por entonces, muchos políticos e intelectuales percibieron al sector como un tipo particular de "industria industrializante", es decir, un ámbito idóneo para potenciar la transferencia de tecnología, formar recursos humanos altamente capacitados y dinamizar a la industria en general (Bernis, 1971; Harriague, Quilici y Sbaffoni, 2008). 
Los procesos de innovación en Argentina -entendidos como la incorporación de conocimiento para modificar la estructura productiva en relación a las necesidades propias de cada sociedadhan resultado dispersos, aislados y desconectados del resto del entramado económico (Azpiazu y Nochteff, 1994; Botana y Sabato, 1975; Hurtado, 2010; Oteiza, 1992; Thomas, Santos, y Fressoli, 2013). En este panorama, el Programa Nuclear Argentino (en adelante PNA) ha constituido una excepción notable a la regla, dado que adoptó el dominio de una tecnología compleja cuyas aplicaciones civiles fueron históricamente monopolizadas por países desarrollados. A contraluz del contexto nacional y latinoamericano, el PNA logró articular políticas públicas de gran continuidad que aseguraron altos presupuestos para el sector y, en consecuencia, permitieron el desarrollo local de ciencia y tecnología sumamente compleja con inserción en el mercado internacional. En este sentido, el accionar estatal articulado mediante la Comisión Nacional de Energía Atómica (en adelante CNEA) resultó preponderante, dado que la institución fue políticamente respaldada por gobiernos de distinto signo y disfrutó de una importante cuota de autonomía.

Ahora bien, los años formativos de la CNEA se enmarcaron en un contexto más general de maduración en la producción manufacturera local relativa a la segunda etapa de la industria sustitutiva (1955-1976), que privilegiaba el fortalecimiento de las industrias metalmecánica y electrónica y otorgaba un importante papel a la inversión extranjera. En esos años también cobró relevancia la discusión sobre la ciencia y la tecnología como componentes estratégicos para fomentar el desarrollo económico y terminar con los lazos de dependencia hacia los países centrales (Dagnino, Thomas y Davyt, 1996). Esta corriente de pensamiento agrupaba intelectuales latinoamericanos de raíz cepalina y/o dependentista que encontraban en el Estado un actor indispensable para materializar (o alentar) el desarrollo económico $11 \mathrm{Al}$ calor de aquellos debates, dentro de la cNEA se consolidaba una "filosofía" o "mística institucional" compartida por la mayoría de sus miembros en torno al concepto de "autonomía tecnológica" (Fernández, 2014). Según su autor intelectual, Jorge Sabato, la noción remitía a fortalecer la adquisición de conocimientos y competencias locales a través de la resolución de los problemas prácticos propios de cada región. De esta forma, se buscaba incrementar la capacidad de decisión respecto de aquello que convenía desarrollar y producir localmente, qué tecnologías importar y bajo qué condiciones asociarse. Es decir, no se trataba exclusivamente de promover tecnologías desarrolladas localmente -interpretación alternativa del concepto que también tuvo difusión dentro de CNEA- sino de definir paquetes tecnológicos sustentables -importados o no- que se adecuaran a las condiciones económicas y sociales preexistentes (Sabato, 1983).

Asimismo, alcanzar la "autonomía tecnológica” requería del apoyo de infraestructura propia capaz de responder a las problemáticas del sector y por este motivo resultaba imperativo que los procesos de innovación desarrollados en la CNEA se derramaran a la industria local a través del "aprender haciendo" (learning by doing), es decir, la resolución de problemas prácticos característicos de la región (Sabato, 1973, p. 110).

Tal y como lo plantea Evans (1995), allí dónde se verifica la escasa vitalidad del capital privado, el Estado es capaz de asumir el rol de "partero" y fomentar la aparición de nuevos grupos empresariales o induciendo a los existentes a aventurarse en rubros más riesgosos. En la CNEA, dicha estrategia transformó a las empresas nacionales en un actor privilegiado de la política oficial y

\footnotetext{
${ }^{1}$ Esta corriente -denominada PLACTED con base en el libro compilado por Jorge Sabato titulado Pensamiento Latinoamericano en Ciencia, Tecnología y Desarrollo-, agrupaba intelectuales como Oscar Varsavsky, Amílcar Herrera, Osvaldo Sunkel, Enrique Oteiza, Francisco Sagasti, Máximo Halty Carrere, entre otros (Sabato, 2011).
} 
se materializó en mecanismos de derrame de tecnologías gestionados a través del Departamento de Metalurgia (1955), el Servicio de Asistencia Técnica a la Industria (1962) y la construcción de las Centrales Nucleares Atucha I (1974) y Embalse (1984) bajo la figura de "apertura del paquete tecnológico" 2 Así, los conocimientos desarrollados por la institución o adquiridos en el exterior fueron ofertados a la industria con el objetivo de fortalecer la provisión de bienes y servicios para el PNA (Enriquez, 2013, p. 21).

Ahora bien, la filosofía institucional de CNEA alcanzó su cenit en un contexto muy particular, signado por la instauración de una dictadura militar (1976-1983) que implementó políticas monetaristas de apertura económica - el "Enfoque monetario de la Balanza de Pagos"- 3 y contribuyó a desmantelar la industria sustitutiva. Al contrario de los lineamientos económicos más generales, durante esos años, el gobierno formuló el primer Plan Nuclear Argentino que concebía la construcción de cuatro centrales -sumadas a las dos ya existentes- hasta el año 2000, la instalación de facilidades para obtener el dominio total del ciclo de combustible y el derrame tecnológico a la industria nacional a fin de transformarla en la principal proveedora de servicios y suministros para las usinas. Adicionalmente, y con el objetivo de facilitar el proceso de aprendizaje de las empresas, la legislación contemplaba conformación de un Arquitecto Industrial (AI) ${ }^{5}$ a partir de la asociación entre CNEA y la empresa extranjera seleccionada para aportar la tecnología. Para 1980 se creó la Empresa Nuclear Argentina de Centrales Eléctricas S. A. (en adelante Enace S. A.) como consorcio entre la CNEA y Siemens Kraftwerk Union (KWU). A través de esta experiencia que reunía al comprador y al vendedor en una firma joint venture, se esperaba que la CNEA operara la transferencia de tecnología y el know how necesarios para reemplazar completamente al diseñador en un lapso de quince años.

Este artículo propone ofrecer un primer acercamiento a un tema casi inexplorado, a saber, la cuestión del fomento a la industria local operada en torno a la firma ENACE, así como a los avatares sufridos por la empresa tras la formulación de políticas públicas de distinto signo y en el marco de un notorio desplazamiento de la economía hacia un modelo de valorización financiera. Por ello, se intentará ofrecer una mirada del problema desde la perspectiva de la historia económica y, más puntualmente, de la vinculación entre el Estado y las empresas. En América Latina, dicho corpus se originó en las décadas de 1960 y 1970 y ha tendido a centrarse en el rol desempeñado por el mundo empresario en el proceso de industrialización sustitutiva. A partir de aquellas investigaciones, resulta cada vez más evidente que los patrones de desarrollo vigentes durante el siglo xx no pueden comprenderse por fuera de las relaciones entre el gobierno y las empresas (Evans, 1995;

\footnotetext{
${ }^{2}$ La adopción del modelo de compra de las Centrales llave en mano con "apertura del paquete tecnológico" apuntaba a desagregar los componentes del paquete para maximizar la participación de la producción nacional (Enriquez, 2013, p. 18).

${ }^{3}$ Según aquella filosofía, la reducción de la inflación sería posible una vez que se procediera a la apertura de la economía y, como consecuencia del incremento de la competencia, de esta forma los precios locales convergerían con los internacionales (Canitrot, 1980, p. 472).

${ }^{4}$ Según la orEa, hasta comienzos de los años setenta, el ciclo del combustible nuclear incluía toda la secuencia de procesos que atravesaba el combustible nuclear: la extracción, el tratamiento y la conversión del uranio; la fabricación y el enriquecimiento del combustible; la generación de electricidad; la reelaboración y el reciclado del plutonio y el uranio en reactores rápidos, y la evacuación definitiva de los desechos procedentes de las plantas de reelaboración (Semenov y Oi, 1993, p. 2).

${ }^{5} \mathrm{El}$ Arquitecto Industrial de una central nuclear es la organización especializada en las tareas de planificación, ingeniería y gestión de la construcción de instalaciones industriales complejas, a cargo de la coordinación de las múltiples actividades del Proyecto (Bertoni, Bogdanowicz, Godoy, Huber, Rudelli, y Solanilla, 2004, p. 11).
} 
Schneider, 1999). Sin embargo, la bibliografía sobre el de análisis de casos de empresas públicas argentinas sigue resultando relativamente acotada (Belini y Rougier, 2008; Castellani, 2018; Regalsky y Rougier, 2015).

Asimismo, cabe destacar que la perspectiva de la historia económica ha estado notoriamente ausente en el análisis del sector nuclear, incluso, en los países desarrollados (Rubio-Varas y De la Torre, 2017). En términos generales, la bibliografía producida al respecto proviene del ámbito de los estudios sociales de la ciencia y la tecnología, las relaciones internacionales o las historias institucionales producidas por los actores que han tenido una participación directa en el surgimiento de los distintos programas nucleares. De esta forma, no resulta sorprendente que, a pesar del carácter excepcional y estratégico que adquirió el PNA desde sus orígenes, solo en los últimos años ha sido incorporado al debate historiográfico local (Hurtado, 2009, 2010, 2014; Lugones, 2015; Marzorati, 2012; Rodríguez, 2014, 2015a, 2015b, 2019). Mientras que la política de derrame tecnológico operada a través del Departamento de Metalurgia y elServicio de Asistencia Técnica a la Industria (SATI) ha sido objeto de estudio de tecnólogos, historiadores de la ciencia y algunos miembros del personal de CNEA, los trabajos que mencionan ciertos aspectos de la trayectoria de la firma Enace S. A. aún resultan escasos (Bertoni, 2012; Enriquez 2013; Hurtado, 2009, 2014; Quilici, 2008, 2010; Rodríguez, 2019).

En la primera parte del trabajo se analizan las consideraciones presentes al momento de concebir el diseño del AI, así como las discusiones que giraron sobre su estructuración. Posteriormente, se reseñan los hitos más importantes de la historia de ENACE con especial énfasis en las dificultades de índole presupuestaria y burocrática que atravesó desde su concepción en 1979 hasta la disolución en 1996. Finalmente, esbozaremos algunas reflexiones y preguntas de investigación a fin de propiciar el debate en el futuro.

\section{El surgimiento de Enace en el marco del Plan Nuclear de 1979}

La detonación india de 1974 inauguró una de las etapas más restrictivas en materia de transferencia de tecnología nuclear a nivel internacional. El hecho no solo reavivó los temores sobre un posible holocausto nuclear, sino que, además, podía interpretarse como el surgimiento de futuros competidores del suministro en la periferia. En consecuencia, en 1975 el "Club de Londres" -o Grupo de Abastecedores Nucleares- impulsó el diseño de políticas comunes de restricción de transferencia de tecnología. Este cambio de postura -impulsado por Estados Unidos y la URSSse justificaba con el argumento de que ciertas etapas del ciclo de combustible podrían constituir la antesala del arsenal atómico: la reelaboración o el reprocesamiento de combustible irradiado; el enriquecimiento de uranio, y la producción de agua pesada. La medida, de carácter retroactiva, obligaba a los países miembros a solicitar autorización previa del Organismo Internacional de Energía Atómica (OIEA) para exportar o reexportar elementos contenidos en la "lista detonadora". A su vez, prohibía la cooperación con aquellos países que no aceptaran las salvaguardas en sus instalaciones (Castro, 1991, p. 307). Más allá del discurso antibelicista -elaborado por aquellos países que, no casualmente, detentaban el monopolio del arma atómica-, la política empleada se orientaba a defender la hegemonía estadunidense sobre el uranio enriquecido a través del desmantelamiento 
del desarrollo tecnológico de combustibles alternativos $\sqrt[6]{6}$ En este sentido, la construcción discursiva de la proliferación jugó un papel central, en tanto fue esgrimida por las potencias como excusa para frenar el avance de los programas nucleares en la periferia (Hurtado, 2014, p. 16).

Entre 1977 y 1980, las presiones externas en contra del desarrollo autónomo argentino constituyeron un poderoso condicionante de las políticas implementadas en el nivel doméstico. Dichos eventos confluyeron con un gobierno dictatorial que -al contrario de los lineamientos económicos y políticos más generales- respaldó activamente el desarrollo del programa nuclear autónomo diseñado por CNEA. Como resultado, en la medida en que la tensión recrudecía, la Cancillería argentina reafirmaba la política de rechazo a la firma del Tratado de No Proliferación 7 y la vocación por el dominio local de todas las etapas del ciclo de combustible.

Con base en esos lineamientos, en enero de 1979 el Poder Ejecutivo Nacional aprobó mediante el Decreto 302/79 el primer Plan Nuclear Argentino. El documento se basaba en las conclusiones generales de la Comisión Interministerial, que, a grandes rasgos, oficializaban los objetivos que la CNEA pergeñaba desde la década anterior: la instalación de cuatro centrales nucleares de 600 Mwe. de potencia, la ratificación de la elección de la línea de uranio natural y agua pesada, 8 un amplio programa de exploración y explotación de los recursos uraníferos en el país y la fabricación de elementos combustibles y agua pesada a nivel industrial. Es decir que el objetivo final consistía en ampliar el parque nucleoeléctrico y lograr la autonomía completa en todas las etapas del ciclo de combustible, desde la explotación del uranio hasta el tratamiento de los materiales radioactivos (Rodríguez, 2014, p. 32).

Paradójicamente, el lanzamiento del Plan coincidía con una serie de políticas implementadas desde el Ministerio de Economía que apuntaban al achicamiento del Estado a través de la "privatización periférica” y la destrucción del entramado industrial como mecanismo para desactivar la conflictividad política (Belini y Rougier, 2008; Canitrot, 1980; Schvarzer, 1987). Sin embargo, la misma "tecnocracia" que implementó medidas de corte neoliberal encontró en la obra pública una poderosa herramienta para concretar la alianza con dos actores clave: por un lado, los grupos económicos que resultaban sumamente beneficiados de los contratos con el Estado y las políticas

\footnotetext{
${ }^{6}$ Además de limitar las exportaciones de uranio enriquecido, Estados Unidos intentaba detener la difusión de otros combustibles alternativos: mientras que la técnica de reprocesamiento permite obtener plutonio como eventual sustituto del uranio, el agua pesada resulta indispensable para los reactores de uranio natural.

${ }^{7}$ Tras la detonación China en 1964, bajo los auspicios de Estados Unidos y la Unión Soviética, se abrió a la firma el Tratado de No Proliferación Nuclear (en adelante TNP). En virtud del mismo, los países miembros se dividirían en dos grupos, a partir de los cuales les corresponderían obligaciones diferenciadas: por un lado, aquellas naciones que hasta el momento no habían desarrollado armas nucleares tendrían prohibido fabricarlas, adquirirlas o almacenarlas, mientras que se les restringía el acceso a material fisionable; por otra parte, aquellos países que ya poseían armamento explosivo -Estados Unidos, Unión Soviética, Reino Unido, Francia y China - se comprometían a promover el uso pacífico de la actividad y llegar a un acuerdo para detener la carrera armamentística y, eventualmente, el desarme general (Castro y Takacs, 1991, p. 32). La cuestión se tornó especialmente espinosa en torno a la tecnología de enriquecimiento de uranio.

${ }^{8}$ Por entonces, las opciones disponibles en materia de reactores nucleares quedaban limitadas al empleo de uranio enriquecido como combustible y agua liviana en el moderador o uranio natural y agua pesada. Mientras que el primero trabajaba con un diseño más moderno, más pequeño y más barato, la cuestión se tornaba complicada en torno al abastecimiento. En este sentido, producir el combustible en el país no era una alternativa viable, no sólo porque la tecnología de enriquecimiento tardaría años en desarrollarse y requería grandes desembolsos de capital, sino que además estaba sometida al sistema de salvaguardas. Como corolario, optar por uranio enriquecido significaba estrechar la dependencia con el único proveedor factible del suministro: Estados Unidos. El uranio natural, en cambio, requería de un aumento considerable de los costos de instalación, pero permitía emplear uranio obtenido y envasado localmente, a la vez que resultaba factible adquirir el know how para producir agua pesada (Sabato, 1970, p. 69).
} 
de promoción industrial; por otra parte, una importante facción de las Fuerzas Armadas que consideraban el fomento de la infraestructura energética como un elemento prioritario en materia de desarrollo y defensa nacional (Castellani, 2009, p. 147) $9^{9}$ En lo sucesivo, el respaldo incondicional otorgado por el gobierno dictatorial en materia de relaciones internacionales y planificación se justificaba por el potencial eléctrico, económico y geopolítico del sector, aún cuando el resto de la economía fuera sometida al ajuste y al avance de la "privatización periférica” (Rodríguez, 2019).

Además de los lineamientos estrictamente energéticos, el Plan Nuclear de 1979 identificaba dos objetivos vitales desde el punto de vista estratégico: la participación de la industria nacional y la consolidación del aparato científico-tecnológico. En cuanto al primer objetivo, el decreto establecía la necesidad de plantear un régimen de promoción de la industria para incrementar su participación en forma progresiva en el PNA. Para este fin, se establecía un cronograma escalonado de instalación de las cuatro Centrales que pretendía evitar tanto la saturación productiva como la dispersión de las capacidades adquiridas (véase cuadro 1). El objetivo final consistía en incrementar la cantidad de servicios y suministros adquiridos en el país, los cuales, en virtud de la política de apertura del paquete tecnológico, habían representado alrededor de un $38 \%$ en la Central Atucha I y $60 \%$ en la Central Embalse de Rio Tercero (Solingen, 1993). Al respecto, cabe aclarar que, dado el tamaño y las características del sector nuclear local, se estimaba que la industria argentina no podría bajo ningún punto de vista exceder el $80 \%$ de participación en las obras. La limitación se debía fundamentalmente a que no existía un mercado interno lo suficientemente amplio para justificar la producción de determinados componentes de alta complejidad relativos a la isla nuclear 10

\footnotetext{
${ }^{9}$ Desde principios de siglo se habían configurado ciertos sectores dentro de las Fuerzas Armadas que concebían que la inversión en infraestructura energética, en tanto sector estratégico de la economía, debía desempeñar un papel fundamental (Rodríguez, 2014, p. 35). Ello explica que durante la última dictadura -y en contraste con otras ramas de la industria- la planificación de grandes obras eléctricas no se manejó con un criterio "eficentista" o privatista, sino que involucró grandes partidas presupuestarias, cercanas al 50 \% de la inversión pública nacional (Carciofi, 1990, p. 31).

${ }^{10}$ Entrevista realizada al Dr. en química Carlos Aráoz (Universidad de Buenos Aires), quien se ha desempeñado como Gerente de Combustibles y Gerente de Tecnología de la CNEA, entre otros cargos (Aráoz, 2017).
} 


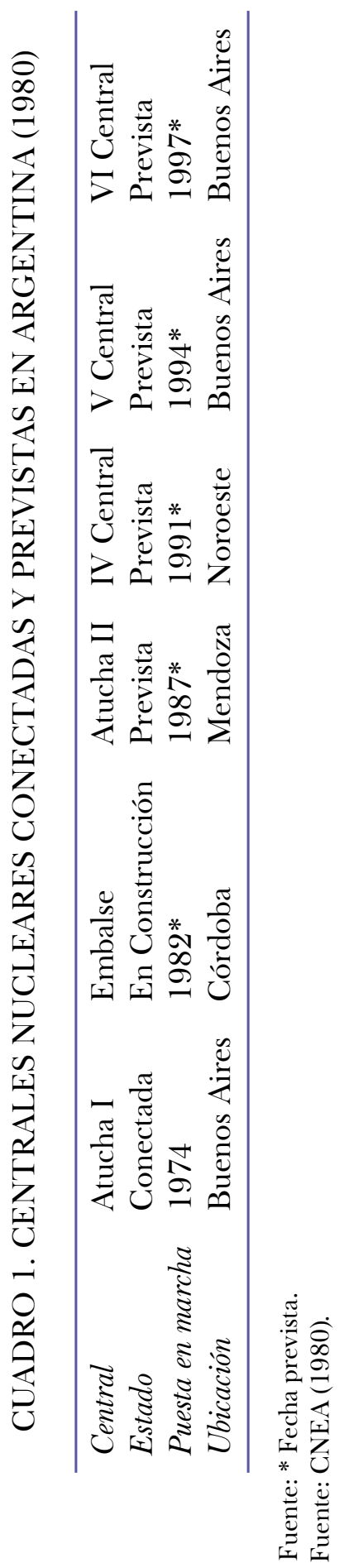


El segundo objetivo estratégico se orientaba a fortalecer las capacidades científicas y tecnológicas en materia nuclear, así como también maximizar el aprovechamiento de los recursos naturales del país. Por este motivo, una parte importante del programa reglamentaba la promoción de la investigación y el desarrollo, la capacitación del personal, la prospección de recursos uraníferos y el fortalecimiento del intercambio científico y tecnológico con el resto de los países de Latinoamérica.

Si bien el Plan Nuclear no especificaba a través de qué mecanismos se lograría transferir la tecnología desde el proveedor al ámbito nacional, desde 1977 circulaba en CNEA la idea de crear una empresa mixta con participación nacional para desempeñar el rol de AI. En este aspecto, se disputaban varios proyectos. Para algunos miembros de la institución, como el Ingeniero Jorge Cosentino, la solución sería otorgar mayor flexibilidad operativa a la Dirección de Centrales Nucleares -en la cual se desempeñaba como gerente-, a través de la conformación de la Empresa Nuclear Argentina S. E. (ENASE). Dicha firma se agruparía en un consorcio con compañías de ingeniería privadas -denominado Argentinatom- con el objetivo de constituir un AI nacional que combinara los conocimientos acumulados en CNEA con el régimen operativo de las empresas. Esta entidad, de carácter estatal, sería la responsable de absorber la tecnología transferida desde el proveedor del diseño al país. En forma alternativa, se barajaba la posibilidad de crear una empresa mixta de tipo joint venture entre el ganador de la licitación de Atucha II y CNEA. La firma, denominada Empresa Nuclear Argentina de Centrales Nucleares S. A. (ENACE), poseería una participación accionaria del $75 \%$ a favor de CNEA y el porcentaje restante sería adquirido por el oferente. El esquema favorecería el entrenamiento del personal, la colocación de una mayor cantidad de pedidos de ofertas en la industria local y la adquisición directa del know how de la ingeniería y el diseño (Bertoni, 2012; Cosentino, 1983, p. 43). En 1979, la discusión, que tuvo lugar al interior de la Dirección de Centrales Nucleares, fue zanjada a favor de la segunda opción.

Ahora bien, en el corto plazo resultaba necesario definir un socio comercial para la construcción de Atucha II -la primera de las Centrales programadas- y la Planta de Agua Pesada. Así, las actividades que se sucedieron en la segunda mitad de 1979 giraron en torno a complejas negociaciones con los proveedores internacionales en el marco del endurecimiento de la política nuclear estadunidense. El proceso de licitación -que básicamente enfrentaba el modelo caNDu de AECL (Canadá) y un prototipo diseñado por Siemenes kwu (República Federal Alemana)- otorgó a la cuestión política un peso fundamental, incluso por encima de consideraciones económicas y tecnológicas. A pesar de que los argumentos a favor del cANDu resultaban más convincentes respecto del criterio de "autonomía tecnológica", la poca disposición del gobierno canadiense para sortear el TNP frustró cualquier posibilidad de optar por esa línea. Finalmente, el 2 de octubre de 1979 el Poder Ejecutivo dio a conocer la elección de KWU como socio comercial para proveer la tecnología.11

\footnotetext{
${ }^{11}$ El 23 de abril de 1979, CNEA había recibido seis ofertas: la propuesta de AECL, que podría ser complementada con el consorcio CANATOM-General Electric (1) o con la firma italiana NIRA (2); dos propuestas de Siemens KWU (3 Y 4) (una con tubos de presión, otra de recipiente de presión). Respecto a la planta de agua pesada, el primer paquete de propuestas se complementaba con la oferta de Lumus (5), mientras que en el segundo caso las facilidades podrían ser provistas por Sulzer Brothers o UHDE (6), empresa en la cual Siemens poseía participación accionaria (La Opinión, 2 de septiembre, 1979).
} 
En este contexto nació la Empresa Nuclear Argentina de Centrales Eléctricas S. A. (EnAce S. A.), constituida el 3 de Noviembre de 1980 con una composición accionaria de 1600 millones de pesos repartidos entre CNeA (75\%) y Siemens KwU (25\%). ${ }^{12}$ Su propósito consistía en efectuar todos los servicios de ingeniería necesarios para llevar a cabo los Proyectos de construcción de Centrales utilizando la tecnología y las licencias del reactor prototipo MzFr alemán, así como el know how en el diseño y construcción de reactores presurizados de agua liviana (PHWR). La empresa debía lograr la capacidad de asumir plenamente las funciones de contratista principal, el manejo de infraestructura, la planificación, el diseño y la dirección del Proyecto. Es decir que, a través de esta experiencia, la CNEA absorbería los conocimientos necesarios para reemplazar completamente al diseñador en un lapso de quince años en forma tal que la participación de KWU en ENACE se reduciría al $20 \%$ en 1985, al $10 \%$ en 1988, y para 1992 la CNEA adquiriría el $100 \%$ de las acciones (Backhaus, 1985, p. 186).

La transferencia de tecnología operaría, fundamentalmente, por medio del training on the job, es decir, el intercambio de comisiones técnicas compuestas por personal argentino y alemán.13 Sin embargo, ciertas cuestiones relativas a la organización y las incumbencias de la entidad no habían sido claramente especificadas. De esta forma, los primeros meses de trabajo en el Proyecto Atucha II comenzaron a poner en evidencia la superposición de tareas entre la CNEA y el AI. Dado que un importante número de miembros del personal jerárquico ocupó puestos en el organismo estatal y la empresa mixta en forma simultánea, se daban situaciones en las que el representante del comprador del Proyecto (CNEA) y el constructor (ENACE) eran literalmente la misma persona. 14 Como consecuencia, se yuxtaponían los equipos de trabajo y las responsabilidades quedaron poco claras.

La situación se resolvió a principios de 1981 a través de la división total de funciones entre la Dirección de Centrales Nucleares -entidad responsable de la compra- y EnACE -contratista principal-. La restricción para ocupar cargos en simultáneo implicó dos dificultades: primero, porque el AI experimentó una significativa disminución del personal técnico argentino, el cual constituiría la base para la recepción de la tecnología alemana; en segunda instancia porque dicha tendencia implicaba la contratación de una mayor cantidad de horas de trabajo en el extranjero. Por último, se decidió que la administración financiera y los contratos quedaran en manos de CNEA, lo que hizo que Enace quedara sin ningún tipo de autonomía y en un plano de subsidiaridad (Bertoni, 2012, p. 6; Cosentino, 1983, p. 44; ENACE, 1983).

La indefinición respecto de la estructuración de ENACE en cuestiones clave puso de manifiesto algunas de las limitaciones operativas de la CNEA al encarar actividades productivas. La rigidez burocrática y la dilación de los tiempos para la conformación del Ai constituyeron síntomas en este sentido. Por otra parte, al optar por la figura de joint venture, también intervinieron los intereses de KWU que, bajo una lógica empresarial, apuntaba a desvincularse de futuras responsabilidades para

\footnotetext{
${ }^{12}$ Boletín Oficial (3 de noviembre de 1980). Estatuto de Empresa Nuclear Argentina de Centrales Eléctricas S. A. Boletín Oficial, Sección 2 (24536), p. 1.

${ }^{13}$ La CNEA designó al Ingeniero Jorge Cosentino (Jefe del Grupo de Reactores Nucleares, quien se había destacado en la dirección del Proyecto Atucha I) como Presidente de la institución, acompañado de un Directorio compuesto por los Jefes de las siguientes reparticiones: la Dirección Técnica (DT), ocupada por el Ing. Etzel; la Dirección Comercial (DC), ocupada por el Ing. Quihillalt, hijo; la Dirección de Asistencia (DA), a cargo del Ing. Pinasco, y la Dirección de Promoción Industrial (DPI), ocupada por el Ing. Aguirre (ENACE, 1981-1982).

${ }^{14}$ A modo de ejemplo, el Ingeniero Cosentino mantuvo durante ese período su cargo como Director del Departamento de Centrales Nucleares (ENACE, 1981-1982).
} 
optimizar las ganancias. Esta situación, sumada al deterioro que la economía nacional experimentó tras la restricción externa posterior a la crisis de 1981,15 constituyen elementos clave para explicar los primeros retrasos del Plan Nuclear.

\section{La empresa Enace y los PRimeros años del Proyecto Atucha II}

A fin de promover el desarrollo de la industria local, se incorporó a ENACE una dirección de promoción industrial que funcionaría como sucesora del Grupo Industria Naciona ${ }^{16}$ creado para Atucha I. Su objetivo era realizar la calificación e integración de las empresas, así como gestionar los contratos firmados con Siemens. Por consiguiente, el AI se transformaba en un nodo clave para facilitar el proceso de transferencia de tecnología, el cual operaría fundamentalmente a través de: 1) la adquisición de licencias de fabricación y la transferencia de tecnología operada desde el exterior; 2) la compra e instalación de equipamiento, y 3) la capacitación en el exterior del personal para su desempeño en el área nuclear.

Respecto al primer punto, se trataba, principalmente, de aquellos conocimientos y licencias provistas por KWU y otras compañías extranjeras para la fabricación de componentes pesados. En esos años, EnACE gestionó la adquisición de tecnología para fabricar dos generadores de vapor, un presurizador, tres enfriadores del moderador y la esfera de contención ${ }^{17}$ (La Opinión, 14 de febrero, 1981). El know how -obtenido a través de la transferencia de documentos, así como del envío de especialistas a Alemania Federal- fue cedido gratuitamente a firmas locales. Adicionalmente, la normativa de garantía de calidad aplicable al proyecto de la central nuclear de Atucha II requería de la presencia de ingenieros en soldadura para certificar la fabricación de los componentes nacionales. A pesar de que en el país podían encontrarse profesionales con amplia experiencia en la materia, no existían centros universitarios que ofrecieran una formación sistemática. ${ }^{18}$ Para resolver dicha dificultad, en 1981 EnACE organizó, junto con el Instituto Alemán de Soldadura, el dictado de un curso en Duisburg (Alemania Federal) destinado a la formación de especialistas. La política fue complementada con el Primer Curso de Ciencia y Tecnología de la Soldadura realizado en Cnea al año siguiente (Asociación Argentina de Soldadura, 2012).

Si bien carecemos de documentos oficiales con información cuantitativa que nos permitan arribar a conclusiones más generales, dicho modus operandi puede verificarse por medio de fuentes indirectas que permitieron identificar algunas de las principales empresas beneficiarias de los mecanismos de transferencia de tecnología. En general, los datos expuestos en el cuadro 2 aunque de carácter acotado y provisorio-, permiten inferir que la transferencia de licencias y la formación de personal en el exterior constituyeron las herramientas más difundidas para la adquisición de know how. El caso más emblemático es el de Industrias Pescarmona (en adelante IMPSA) y

\footnotetext{
${ }^{15}$ En 1981, la política del "Enfoque Monetario de la Balanza de Pagos” y la estatización de la deuda desataron una debacle económica y bancaria que aceleró sustancialmente el proceso de endeudamiento de las empresas públicas.

${ }^{16}$ El Grupo Industria Nacional fue creado en 1965 con la finalidad de relevar las capacidades existentes en el país para determinar la cantidad de suministros que podían ser licitados localmente para la Central Atucha I.

${ }^{17}$ Los generadores de vapor tienen la misión de trasladar el calor generado en el circuito primario al circuito secundario del reactor; el presurizador y los moderadores se encargan de moderar la presión y la temperatura del agua pesada (La Opinión, 14 de febrero, 1981). La esfera de contención es una semiesfera de hormigón que recubre a todo el reactor. En el caso de Atucha II, mide 56 metros de diámetro y pesa 2450 toneladas (Zunino, 1982).

${ }^{18}$ De hecho, en 1977 se había fundado la Escuela de Soldadura de la Gerencia Servicios para Centrales, a fin de proveer soldadores para el montaje de la Central Embalse. La misma asistió a la elaboración y calificación de los distintos procedimientos de soldadura de AECL, basados en los requisitos de la empresa diseñadora de la planta (NA-SA, 2018).
} 
ha suscitado mayor atención por parte de los investigadores. La firma fue originalmente fundada por Enrique Pescarmona en 1907, en la Provincia de Mendoza, como taller de metalurgia liviana dedicada al mercado interno. A mediados de 1970, sus vínculos contractuales con el Estado y la apuesta a la incorporación de tecnología dieron como resultado un importante despegue que se tradujo en la diversificación de los bienes y servicios ofrecidos y la apertura de nuevos mercados. Por entonces, IMPSA participaba en una gran variedad de proyectos de obra pública del sector energético, como el complejo Futaleufú, Agua del Toro y la Central Embalse. En 1978 -momento en que comenzó a plantearse el Plan Nuclear y el proyecto de ampliación del parque nucleoeléctricocomenzó a incursionar en los aspectos más complejos del sector nucleoenergético con la elaboración de piezas, turbogrupos y trabajos para el recipiente de presión (Gutiérrez, 2013).

Es así que la cesión gratuita de la tecnología y la licencia para fabricar los generadores de vapor y presurizadores de Atucha II, que ENACE adquirió del consorcio KWU-MAN, Voestalpine y del grupo Empresas de Ingeniería Españolas, se tradujeron en la formación de profesionales en el exterior y en la compra de determinados bienes de capital que en un futuro permitirían a IMPSA posicionarse en el mercado internacional (Quilici, 2008, p. 15; Rivelis, 1985). De forma similar, Techint S.A. recibió tecnología provista por KwU para el Proyecto Atucha II, por medio del Consorcio Hochtief-Techint-Bignoli (нтв). La empresa se había desempeñado en el Programa Nuclear con anterioridad como subcontratista de Cometarsa S. A. para las tareas de montaje de Atucha I. Posteriormente, durante la construcción de Embalse, había provisto servicios más complejos ligados a la ingeniería civil de la isla nuclear (Energía Nuclear, 1982).

En estos primeros años del Plan Nuclear se auguró un ámbito de negocios al largo plazo para las empresas comprometidas con las actividades nucleoeléctricas y, en consecuencia, muchas firmas comenzaron a emprender procesos profundos de reconversión con miras a la participación en las centrales proyectadas. Así pues, el sector no solo se transformaba en una oportunidad para el derrame de conocimientos del sector a la industria local, también el cumplimiento de estándares internacionales de calidad permitía a las firmas mejorar la oferta de servicios y suministros producidos. En consecuencia, muchas de las empresas beneficiarias y las disciplinas promocionadas -fundamentalmente, la soldadura- lograron una paulatina inserción en otros grandes proyectos vinculados a la obra pública (Bertoni, 2012, p. 9).

Sin embargo, en la medida en que el proyecto padecía retrasos en la asignación del presupuesto, se visibilizaron dificultades en el orden burocrático. Algunos miembros del directorio de ENACE responsabilizaron a la CNEA por el "retaceo" de personal técnico experimentado necesario para llevar a cabo las tareas de la central Atucha II. Según Cosentino, posterior al reordenamiento administrativo, el AI recibió solo el $50 \%$ del personal requerido, hecho que obligó al Comité Ejecutivo a solicitar recursos humanos adicionales en Alemania Federal (Cosentino, 1983, p. 43; EnACE, 1983). En consecuencia el proceso de transferencia de tecnología se vio seriamente perjudicado. Adicionalmente, la estructura otorgada a ENACE -fuertemente centralizada en la CNEA vía la Dirección de Centrales Nucleares- no estaba dotada de la flexibilidad administrativa necesaria para hacer frente a la gestión de los proyectos. Al poseer la mayoría accionaria del Estado a través de la CNEA, hubo demoras y dificultades derivadas de la Ley de Contabilidad y el extenso ciclo de auditorías que se traducían en el aumento de los costos y el deterioro de la capacidad financiera de las pequeñas y medianas empresas que participaban en el proyecto Atucha II (ENACE, 1981-1982, 1983; Volman, 1986, p. 13). Como resultado de esto, el 23 de junio de 1983 -a solo tres años de iniciado del proyecto- la obra Atucha II ratificaba formalmente un retraso de 24 meses respecto al cronograma original. 


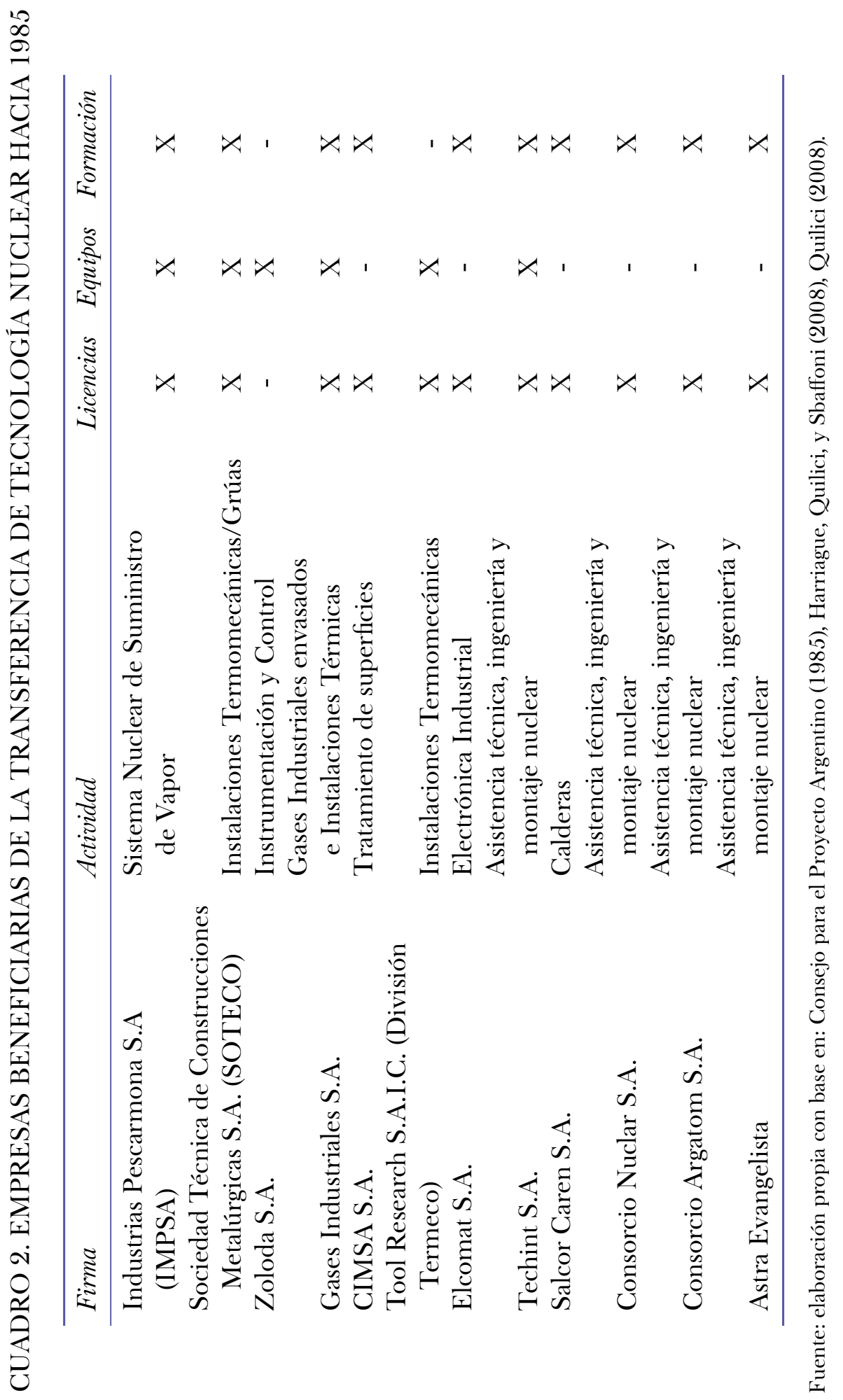




\section{Los años de la democracia Y el Recorte del Plan Nuclear}

Sin duda, las décadas de 1980 y 1990 constituyeron una etapa de declive para la expansión de la nucleoelectricidad en todo el mundo. Intervenidos por dificultades de diversa índole -tecnológicas, económicas, políticas y sociales- los programas nucleares de Occidente atravesaron un período de repliegue significativo que se tradujo en una mayor visibilidad de los movimientos de resistencia a la actividad nuclear ${ }^{19}$ (Castro, 1991, p .110; Castro y Takacs, 1991, p. 12; Piaz, 2015, p. 116). Sin embargo, en América Latina el principal escollo a la continuidad de los programas nucleares provino de la crisis de la deuda y el ahogo financiero provocado por los principales organismos internacionales. En efecto, los gobiernos de corte autoritario instaurados en la década anterior se habían sumado a un vertiginoso proceso de endeudamiento externo, orientado a financiar empresas estatales y grandes obras públicas. Cuando la Reserva Federal de los Estados Unidos impulsó el aumento de las tasas de interés, no solo se produjo una violenta restricción crediticia, sino que obligó a los estados del Cono Sur a suspender los pagos o renegociar sus deudas 20

En este marco se desarrolla el problema de la restricción externa, que constituyó uno de los aspectos más problemáticos de la restauración democrática llevada a cabo por el gobierno del Dr. Raúl Alfonsín (1984-1989). Durante aquellos años, la implementación de políticas económicas dependió en forma directa de la posibilidad de renegociar la deuda externa con los respectivos acreedores y el FMI, así como de la capacidad de contrarrestar el empoderamiento de los principales grupos económicos surgidos durante la última dictadura. El fracaso en ambos frentes contribuyó a profundizar la crisis de la autonomía estatal, de la cual el sector energético no salió indemne. A la vez que la obra pública dejaba de ocupar el papel que desempeñó en el gobierno anterior, la tendencia fue hacia el abandono de las grandes obras eléctricas, el recorte presupuestario de las instituciones y el endeudamiento de las empresas estatales (Azpiazu y Schorr, 2010, p. 114). En el plano nuclear, el ajuste económico se conjugó con el retorno de las presiones internacionales especialmente tras el anuncio de que Argentina había logrado enriquecer uranio- 21 a la vez que la vinculación histórica entre la CNEA y las Fuerzas Armadas arrojaban una sombra de duda frente al proceso de enjuiciamiento de los responsables de la violación de Derechos Humanos. Así fue como la preponderancia del sector nuclear se desintegraba frente a la desmilitarización, el recorte presupuestario y el avance de los movimientos de resistencia (Rodríguez, 2015).

$\mathrm{Al}$ mismo tiempo que se acotaba la proyección de la demanda eléctrica a futuro y se apostaba a la explotación de los recursos gasíferos, la CNEA perdió su lugar privilegiado en la asignación de presupuesto e ingresaba en la puja intersectorial con las demás reparticiones estatales. Sintomáticamente, el Plan Energético Nacional de 1985 reemplazó la proyección de las tres centrales

\footnotetext{
${ }^{19}$ Los movimientos de resistencia cobraron mayor visibilidad tras los eventos de Three Mile Island y Chernóbil. El accidente nuclear de Three Mile Island ocurrió el 28 de marzo de 1979, cuando se produjo un fallo en el circuito secundario del reactor que derivó en la fusión parcial del núcleo. Si bien se trató de un error técnico, resultó agravado por decisiones humanas. El accidente de Chernóbil ocurrió el 26 de abril de 1986, tras una prueba en el núcleo que explotó la tapa del reactor produciendo el incendio de la planta y emitiendo gases tóxicos a la atmósfera. Según la Escala Internacional de Sucesos Nucleares y Radiológicos confeccionada por el OIEA, el primer accidente se clasificó de magnitud 5 y el segundo de 7 .

${ }^{20}$ Según Marichal, el servicio anual de las deudas externas de los países del Tercer Mundo pasó de 20 mil millones de dólares, en 1981, a casi 100 mil millones de dólares en 1982 (Marichal, 2010, p. 209).

${ }^{21}$ El 18 de Noviembre de 1983, el Presidente de Cnea, Carlos Castro Madero, publicó la noticia de que Argentina adquirió la capacidad de obtener uranio enriquecido a escala piloto mediante el sistema de difusión gaseosa, a través de las facilidades instaladas en secreto en Pilcaniyeu, a 70 kilómetros de Bariloche (La Razón, 19 de noviembre, 1983).
} 
nucleares restantes por dos módulos de mediana capacidad (350 Mw.) ${ }^{22}$ de los cuales el gobierno nunca llegó a definir mayores especificaciones (Subsecretaría de Planificación Energética, 1986). Así, el propósito inicial de la firma ENACE -concebida como el nexo para el know how adquirido en el exterior y las empresas locales- se tornó difuso. La decisión de reemplazar las usinas previstas por módulo de pequeña potencia, y la paralización progresiva de los trabajos en Atucha II -fundamentalmente, a partir de 1988-, arrojaban un velo de incertidumbre frente a las actividades futuras de la empresa y la viabilidad del sector como ámbito de negocios en el largo plazo. Dada la contracción de la actividad, ENACE ofreció sus servicios como AI para la industria convencional, sobre todo en algunas reparticiones estatales como SEgBA, Astilleros Domecg García y Agua y Energía Eléctricas (Argentina Nuclear, 1987, p. 28). Sin embargo, dicha diversificación resultó acotada, en tanto que la empresa -claramente orientada a satisfacer demandas estatales- carecía de un departamento de ventas (SIGEN, 1994).

Desde el punto de vista nuclear, durante esos años el único proyecto de envergadura -a excepción de los trabajos para Atucha II- fue el diseño del reactor Argos 380, impulsado por la gestión de Abel González en Enace. Dado que el Plan Energético preveía la instalación futura de dos módulos de $350 \mathrm{Mw}$., se diagramaron dos prototipos nacionales capaces de responder a esa demanda con alto grado de flexibilidad en el equipamiento combustible. El diseño de CnEa fue nombrado TPA 300 y EnACE hizo lo propio con el ARGOS 380. Sin embargo, en el corto plazo ninguno de los dos proyectos recibió respaldo oficial, a la vez que el gobierno nunca llegó a definir políticas más concretas para la expansión del parque nucleoeléctrico. Así pues, a partir de 1986 comenzó a considerarse la posibilidad de privatizar ENACE como iniciativa encuadrada en una política más general de reducción del déficit fiscal impulsada por el Plan Austral ${ }^{23}$ La venta a manos privadas era entendida como una "solución" frente a la necesidad de aumentar la rentabilidad, agilizar la estructura burocrática y aliviar las cuentas estatales.

Dicha propuesta suscitó el debate en el seno de CNEA, a pesar de que el directorio matizaba la idea de un simple traspaso de empresas a manos privadas. Según Emma Pérez Ferreira, entonces presidenta de la institución, se trataba de promover un carácter empresarial al manejo de las actividades productivas, pero sin separarla tajantemente de la órbita de CNEA. Frente a esta posibilidad, Abel González planteaba como alternativa la distribución del paquete accionario entre sujetos con “vocación" por el desarrollo nuclear: entidades públicas, privadas y accionistas individuales provenientes del personal científico. A pesar de que dichas ideas fueron volcadas en un documento emitido por el Consejo Consultivo, en ese entonces el gobierno no adoptó ninguna decisión oficial (CNEA, 1988).

\footnotetext{
${ }^{22}$ Con la compra de las centrales, la CNEA adquirió licencias en Siemens y CANDu para construir reactores de potencia de tubos o recipientes de presión. A pesar del debate suscitado al interior de la institución, respecto a qué tecnología resultaría más conveniente, el gobierno nunca adoptó una postura oficial al respecto.

${ }^{23}$ En 1985, Alfonsín designó a Juan Vital Sourrouille al frente del Ministerio de Economía. La nueva gestión impulsó el Plan Austral que, a grandes rasgos, concertaba el congelamiento de precios con el empresariado local, limitaba el gasto público y reducía la injerencia estatal a través de privatizaciones y el incentivo a las inversiones privadas (Azpiazu y Schorr, 2010, p. 84).
} 
EL PRINCIPIO DEL FIN: EL PROGRAMA DE PRIVATIZACIÓN Y LA LIQUIDACIÓN DE ENACE

La imposibilidad de hacer frente a los compromisos de pago adquiridos por los países Latinoamericanos durante la década de 1980, condujeron al diagnóstico de la "década perdida", motivo por el que gran parte de la comunidad económica internacional de acreedores se reunió en Washington por iniciativa del gobierno de los Estados Unidos (Brieger, 2002). Con este hecho se procedió a la reestructuración de las deudas bajo los postulados del neoliberalismo implementados por el Banco Mundial, el Tesoro de Estados Unidos, el Banco Interamericano de Desarrollo y el Fondo Monetario Internacional. En paralelo, el lanzamiento del Plan Brady logró reconfigurar las relaciones entre la periferia y los acreedores a través de la emisión de bonos del tesoro de los Estados Unidos que no pagaban intereses, sino que se capitalizaban a lo largo del tiempo. En muchos casos, la canalización de una gran masa de activos líquidos se vería asegurado por la adopción del Plan Baker, es decir, la privatización de las empresas públicas (Azpiazu y Schorr, 2010, p. 140; Basualdo 2013, p. 349).

En Argentina, la instauración del orden neoliberal alcanzó su forma más acabada durante las presidencias de Carlos Menem (1989-1994 y 1994-1999). Entre 1989 y 1991 se procedió a la apertura acelerada al comercio internacional y la suspensión de los regímenes de promoción industrial, a la redefinición del papel del Estado con especial énfasis en la descentralización y el achicamiento de sus funciones y a la instauración de la paridad cambiaria ${ }^{24}$ En el ámbito de los servicios públicos, la influencia del "modelo británico'25 configuró un contexto sumamente adverso que se expresó en el proyecto de privatización de empresas públicas lanzado a mediados de la década. A medida que se cimentaron los pilares del orden neoliberal, las presiones externas para la adopción del Plan Baker y el abandono de las pretensiones de "autonomía tecnológica" nuclear se agudizaron notoriamente. En el plano local dicha opinión fue compartida por gran parte del Ministerio de Economía tras el ingreso de Domingo Cavallo 26 y el think tank de la Fundación Mediterránea en 1990. Estos grupos veían con desaprobación las inversiones públicas "de riesgo" desembolsadas para la construcción de las grandes obras del plan nuclear, las cuales solo podían ser política y económicamente redituables en el largo plazo. De esta forma, el proyecto de las privatizaciones resultó funcional para la conformación de la "comunidad de negocios" que reconciliaba

\footnotetext{
${ }^{24}$ Los tres pilares del orden neoliberal fueron establecidos a través de la Ley de Emergencia Económica (23.697), la Ley de Reforma del Estado (23.696) y la Ley de la Convertibilidad (23.928), mediante la cual el Banco Central debía asegurar el respaldo total del circulante en pesos a través de la compra de dólares, de forma tal que un peso equivaldría a un dólar.

${ }^{25}$ Durante las gestiones de Margaret Thatcher (1979-1990) y John Major (1990-1997) en Gran Bretaña, la venta de empresas estatales alcanzó sectores que hasta entonces habían sido considerados estratégicos -como la energía y los ferrocarriles- con la finalidad de incentivar la competitividad y simplificar el proceso de regulación (Thomas, 1999, p. 106). A grandes rasgos, el "modelo" británico se componía de cuatro aspectos: la creación de un mercado spot al por mayor como el principal escenario de fijación de precios para las ventas de electricidad mayoristas; la incorporación de competencia minorista para que todos los consumidores puedan elegir su proveedor de electricidad; la separación corporativa estricta de las actividades que seguirían siendo monopolios (como la transmisión) de aquellas otras que serían impulsadas por el mercado, y la separación corporativa entre la generación y distribución (Thomas, 2004, p. 4).

${ }^{26}$ Domingo Cavallo fue el principal impulsor de la Ley de Convertibilidad. Provenía de las filas del Instituto de Estudios sobre la Realidad Argentina y Latinoamericana (IERAL) de la Fundación Mediterránea, organización de corte liberal fundada en 1977 a partir de un equipo de economistas egresados de la Universidad de Córdoba. Luego de haber obtenido un título doctoral en la Universidad de Harvard, comenzó una larga trayectoria en cargos públicos -incluyendo la Presidencia del Banco Central en 1982 y la asunción como diputado del Partido Justicialista en Córdoba en 1987que alcanzaría su cenit durante la gestión menemista.
} 
los intereses del capital extranjero y los grupos económicos locales. En el caso del sector eléctrico, las reformas aplicadas a partir de 1990 contribuyeron a incrementar el proceso de acumulación de renta extraordinaria por parte de los "ganadores" de la retirada estatal y a acentuar la dependencia energética respecto a las fuentes térmicas convencionales (Schorr, 2004).

En agosto de 1994, para sorpresa de gran parte de la comunidad científico-tecnológica, el Poder Ejecutivo promulgó el decreto 1540/94 con los principales lineamientos para la llevar a cabo la privatización de las centrales. Al igual que en otras ramas del sector eléctrico, la decisión fue tomada unilateralmente por el Poder Ejecutivo, distanciándose de las propuestas de la CNEA y la Secretaría de Energía. En principio, la normativa elaborada por Cavallo y la Fundación Mediterránea reorganizaba las funciones de CNEA en distintas unidades -generación, regulación e investigación- a fin de facilitar el traspaso a la esfera privada. El resultado serían tres instituciones: 1) una sociedad anónima (Nucleoeléctrica Argentina S. A.) con totalidad de acciones en el Estado Nacional que operaría las Centrales Atucha I y Embalse, y se encargaría de la construcción, puesta en marcha y operación de la central Atucha II hasta la privatización; 2) un ente regulador (ENREN) que funcionaría de forma autárquica y se encargaría de fiscalizar y regular la actividad, y 3) la CNEA original, solo retendría las funciones de formación de recursos humanos, investigación y desarrollo de tecnologías (Rodríguez, 2015b).

Inicialmente, en este esquema el destino de ENACE sería el de continuar su operación como arquitecto e ingeniero de las centrales nucleares en construcción, hasta su privatización, en tanto que NASA se volvía propietaria de $75 \%$ de la empresa. Como resultado, el AI fue reestructurado, pasando de tener 700 empleados a 300 (Argentina Nuclear, 1993). Finalmente, la suspensión definitiva del proyecto Atucha II tuvo como consecuencia la liquidación de la institución, luego del vencimiento del estatuto societario en 1996 (EnAce, 1996). Del plantel "superviviente" de 120 personas que quedaban en la obra, solo se conservó un grupo de entre 40 y 50 profesionales bajo la dependencia de NASA, a fin de llevar a cabo tareas de mantenimiento. El resto, fue sometido a la política de retiro voluntario (Clarín, 1 de diciembre, 1996). Así se desintegraban los grupos de trabajo especializados que la CnEA formó a través de la adquisición de know how en el exterior, reduciendo significativamente el acervo de conocimientos acumulado durante las últimas décadas en materia nucleoeléctrica. Al finalizar la vigencia estatuaria de ENACE el 17 de diciembre de 1996, se designó una comisión fiscalizadora que procedió a liquidar la empresa, desmontando así el último pilar que sustentaba la filosofía de "autonomía tecnológica".

\section{Conclusiones}

A todas luces, el contexto internacional restrictivo que se configuró a mediados de la década de 1970 condujo a reforzar el concepto de "autonomía tecnológica" y la vocación por el dominio de todas las etapas del ciclo de combustible. Como resultado, el plan nuclear que surgía en 1979 proyectó la producción local de todos los insumos necesarios -desde la extracción de uranio hasta su reprocesamiento- a través de la adquisición de los conocimientos en el exterior. En este sentido, es claro que el plan de instalación de las centrales nucleares no se planteó únicamente como un programa de obra pública con el objetivo de generar energía. Además de contribuir al sistema eléctrico, las usinas fueron delimitadas como herramientas esenciales para el desarrollo científico

y tecnológico del sector nuclear y el derrame de dichos conocimientos hacia la industria local. Es así 
que Enace S. A. fue concebida como una herramienta clave para operar el proceso de transferencia de conocimientos a través de la formación de recursos humanos en el exterior y la adquisición de equipos y licencias.

La trayectoria de la empresa analizada resulta significativa ya que -más allá de sus límites en términos organizacionales y económicos- se trató de una respuesta local a la problemática específica de la dependencia tecnológica, además logró importantes resultados en materia de adquisición de conocimientos. Mientras que gran parte del know how capitalizado por firmas como IMPSA o Techint sirvieron como base para otras industrias convencionales o pudieron posicionarse en el mercado internacional, los recursos humanos de ENACE, "supervivientes" de la década de 1990, constituyeron la base para el relanzamiento nuclear operado en el 2006 (Rodríguez, 2019). Sin embargo, también es preciso destacar algunas limitaciones de la experiencia. En este sentido, es claro que las estrategias de fomento de la industria local emanadas de CNEA fueron concebidas entre 1950 y 1960 en el marco de un modelo de acumulación de corte desarrollista. De esta forma, los rasgos centrales de la filosofía de "autonomía tecnológica" adquirieron sentido solo en un contexto de profundización de la industria sustitutiva, la cual perdió posiciones frente al avance del proceso de desindustrialización durante la última dictadura y acelerado en las décadas posteriores. Mientras que en la CNEA se buscaba subsidiar y priorizar la compra de suministros producidos localmente, la destrucción de ciertas ramas de la industria planteó a posteriori contradicciones que se materializaron en un "cuello de botella" para las necesidades de las obras proyectadas. Esta tendencia se manifestó con más fuerza luego del retorno de la democracia.

Como corolario -y a pesar de que el concepto continuó jugando un rol clave en la definición de políticas públicas-, los lineamientos de la "autonomía tecnológica” deben ser revisados a la luz de las modificaciones ocurridas en el capitalismo argentino en torno a la transición hacia el modelo de valorización financiera. Dado que constituyó un eje central de la "mística institucional" o identidad de la CNEA, resulta sintomático que muy pocos de sus integrantes problematizaran la cuestión. De esta forma, se abre la interrogante de si las políticas de transferencia de tecnología promocionadas por ENACE no habrían contribuido a profundizar la tendencia a la "patria contratista" en el sector nuclear, la cual comenzaba a ser denunciada desde fines de 1980 (Bertoni, 2012, p. 5; Cosentino, 1983; Hurtado, 2014; Lugones, 2015; Quilici, 2008, p. 16). Sin embargo, aún es poco lo que se ha indagado sobre de la naturaleza de dicha interacción, quedando pendiente para investigaciones futuras el análisis de la naturaleza del vínculo entre el programa nuclear, las centrales y las empresas privadas.

LISTA DE REFERENCIAS

Aráoz, C. (2017, junio 7). Entrevista al Dr. En Química Carlos Aráoz (Entrevista realizada por la autora).

Asociación Argentina de Soldadura (2012, agosto 6). Recuperado de https://www.demaquinasyher. amientas.com/soldadura/asociacion-argentina-de-soldadura

Atucha II se queda sin fondos (1996, diciembre 1). Diario Clarín.

Azpiazu, D. y Nochteff, H. (1994). El desarrollo ausente. Buenos Aires: Ensayos de economía política.

Azpiazu, D. y Schorr, M. (2010). Hecho en Argentina: Industria y economía, 1976-2007. Buenos Aires: Siglo Veintiuno Editores/FLACSO. 
Backhaus, K. (1985). Cooperación argentino-alemana. En Consejo para el Proyecto Argentino. En Consejo para el Proyecto Argentino (ed.), El desarrollo nuclear argentino. Buenos Aires: Edigraf.

Balogh, B. (1991). Chain reaction: Expert debate and public participation in American commercial nuclear power, 1945-1975. Cambridge: Cambridge University Press.

Basualdo, E. M. (2013). Estudios de historia económica Argentina: Desde mediados del siglo XX a la actualidad (3ra.). Buenos Aires: Siglo Veintiuno Editores.

Belini, C. y Rougier, M. (2008). El Estado empresario en la industria argentina: Conformación y crisis. Buenos Aires: Manantial.

Bernis, G. de. (1971). Les industries industrialisantes et les options algériennes. Revue Tiers Monde, 12(47), 545-563. DOI: 10.3406/tiers.1971.1802

Bertoni, J. (2012). Central Nuclear Atucha II. Su Génesis. Buenos Aires: TEA.

Bertoni, J., Bogdanowicz, E., Godoy, A., Huber, H., Rudelli, M. y Solanilla, R. (2004). Proyecto Central Nuclear Atucha II. Para su culminación exitosa. Buenos Aires: s. d.

Botana, N. y Sabato, J. (1975). La ciencia y la tecnología en el desarrollo futuro de América Latina. En J. Sabato (ed.), El Pensamiento Latinoamericano en la problemática Ciencia, Tecnología, Desarrollo, Dependencia (pp. 143-154). Buenos Aires: Edigraf.

Brieger, P. (2002). De la década perdida a la década del mito neoliberal. En J. Gambina (ed.), La Globalización económico-financiera (pp. 341-355). Buenos Aires: CLACSO.

Canitrot, A. (1980). La disciplina como objetivo de la politica económica. Un ensayo sobre el programa económico del gobierno argentino desde 1976. Desarrollo Económico, 19(76), 453475. DOI: $10.2307 / 3466561$

Carciofi, R. (1990). La desarticulación del pacto fiscal. Una interpretación sobre la evolución del sector argentino en las dos últimas décadas. Buenos Aires: Comisión Económica para América Latina y El Caribe.

Castellani, A. (2009). Estado, empresas y empresarios: La construcción de ámbitos privilegiados de acumulación entre 1966 y 1989. Buenos Aires: Prometeo Libros.

Castellani, A. G. y Iramain, L. D. (2017). El deterioro del Estado-empresario: Transformaciones estructurales y desempeño de las empresas públicas argentinas (1976-1983). América Latina en la Historia Económica, 25(2), 239-271. DOI: 10.18232/alhe.866

Castro, C. y Takacs, E. A. (1991). Política nuclear argentina: Zavance o retroceso? Buenos Aires: El Ateneo.

Castro, F. (1991). Energía nuclear y desarrollo: Realidades y desafíos en los umbrales del siglo XXI. Buenos Aires: Ediciones Colihue.

Central Nuclear Atucha II. Continuado el avance para 1993 (1993). Argentina Nuclear, 2, 12-17.

Comisión Nacional de Energía Atómica (1988). Informe del Consejo Consultivo sobre políticas y estructura de la institución. Buenos Aires: Autor.

Cosentino, J. (1983). GNA II y ENACE S. A. Expectativas y realidad de dos decisiones coyunturales. Realidad energética, 41-61.

Dagnino, R., Thomas, H. y Davyt, A. (1996). El pensamiento en ciencia, tecnología y sociedad en Latinoamérica: Una interpretación política de su trayectoria. Redes, 7(3), 13-51.

Empresa Nuclear Argentina de Centrales Eléctricas S. A. (1981). Actas del Comité Ejecutivo (Vol. 1). Buenos Aires: Autor.

Empresa Nuclear Argentina de Centrales Eléctricas S. A. (1983). Actas del Comité Ejecutivo (Vol. 2). Buenos Aires: Autor. 
Empresa Nuclear Argentina de Centrales Eléctricas S. A. (1996). Relevamiento del Estado patrimonial de activos y pasivos ciertos y contingentes al 31/12/96. Buenos Aires.

ENACE S. A. (1987). Argentina Nuclear, 2(9-10), 28-29.

Energía Nuclear (1982). Infraestructura Nuclear del Sector Privado en Argentina. Informe No1: Techint en el desarrollo nuclear argentino. Energía Nuclear, 2(9), 25-29.

Enriquez, S. (2013). La transferencia de tecnología en la CNEA: entre el "ofertismo" y el Plan Nuclear | IADE. Revista de la CNEA, 13(49/50), 17-27.

Evans, P. (1995). Embedded Autonomy. States and Industrial transformations. Nueva Jersey: Princeton University Press.

Exhaustivo informe de ofertas para Atucha II (1979, septiembre 2). Diario La Opinión.

Fernández, A. (2014). Entre la mística y la politización: Análisis de las tensiones interpretativas sobre la memoria institucional de la GNEA (1973). KULA. Antropólogos del Atlántico Sur, (11), 24-40.

Frewer, H. y Altvater, W. (1977). Technology transfer by industry for the construction of nuclear power plants. Annals of Nuclear Energy, 4(6-8), 235-248. DOI: 10.1016/0306-4549(77)900329

Gutiérrez, C. (2013). IMPSA: atractivos y paradojas del éxito tecnoindustrial en la periferia. En H. Thomas, G. Santos y M. Fressoli (eds.), Innovar en Argentina. Seis trayectorias empresariales basadas en estrategias intensivas en conocimiento (pp. 71-104). Buenos Aires: Lenguaje Claro.

Harriague, S., Quilici, D. y Sbaffoni, M. (2008). Estilos socio-técnicos en el sector nuclear argentino. Crisis y sustentabilidad. XXI Jornadas de Historia Económica. Presentado en Caseros. Caseros: Universidad Nacional de Tres de Febrero.

Hurtado, D. (2009). Periferia y fronteras tecnológicas. Energía nuclear y dictadura militar en la Argentina (1976-1983). CTS: Revista iberoamericana de ciencia, tecnología y sociedad, 5(13), 27 64.

Hurtado, D. (2010). La Ciencia Argentina, un Proyecto inconcluso (1930-2000). Buenos Aires: Edhasa.

Hurtado, D. (2014). El sueño de la Argentina atómica. Política, tecnología nuclear y desarrollo nacional (1945-2006). Buenos Aires: Edhasa.

La política nuclear quedará en lo sucesivo bajo el estricto control de Presidente de la República (1983, noviembre 19). Diario La Razón.

Lugones, M. (2015). Politica nuclear y valorización financiera (1976-2003): ל̇hacia una nueva estrategia de desarrollo? Presentado en V Jornadas de Historia de la Industria y los Servicios, Buenos Aires.

Marichal, C. (2010). Nueva historia de las grandes crisis financieras: Una perspectiva global, 1873-2008. Barcelona: Editorial Debate.

Marzorati, Z. del V. (2012). Plantear utopías: La conformación del campo científico-tecnológico nuclear en Argentina, 1950-1955. Buenos Aires: Ediciones CICCUS/CLACSO.

NA-SA. (2018). Una escuela para un oficio-Nucleoeléctrica Argentina. Recuperado de http://www na-sa.com.ar/prensa/una-escuela-para-un-oficio/

Oteiza, E. (ed.). (1992). La Política de investigación científica y tecnológica argentina: Historia y perspectivas. Buenos Aires: Centro Editor de América Latina.

Participación Nacional en el Plan Nuclear (1981, febrero 14). Diario La Opinión.

Piaz, A. (2015). Acciones de resistencia a la tecnología nuclear en Argentina: Mapeando el terreno. REDES, 21(41), 111-140. 
Quilici, D. (2008). Desarrollo de proveedores para la industria nuclear argentina Visión desde las Centrales Nucleares.H-industri@: Revista de historia de la industria, los servicios y las empresas en América Latina, (2), 1-1.

Quilici, D. (2010). La fabricación de los elementos combustibles para los reactores nucleares de potencia en Argentina: Un caso de inversiones productivas realizadas por un organismo de ciencia y técnica. Revista de la CNEA, 23-39.

Regalsky, A. M., Rougier, M. y Salerno, E. (eds.). (2015). Los derroteros del estado empresario en la Argentina: Siglo XX. Buenos Aires: Editorial de la Universidad Nacional de Tres de Febrero.

Rivelis, A. (1985). Grandes Componentes. En Consejo para el Proyecto Argentino (ed.), El desarrollo nuclear argentino (pp. 97-102). Buenos Aires: Edigraf.

Rodríguez, M. (2014). Avatares de la energía nuclear en Argentina. Análisis y contextualización del Plan Nuclear de 1979.H-industri@: Revista de historia de la industria, los servicios y las empresas en América Latina, 8(15), 30-55.

Rodríguez, M. (2015a). La política científica y tecnológica luego del retorno de la democracia y su impacto en la Comisión Nacional de Energía Atómica. En C. Lértora (ed.), Política científica y tecnológica. Estado y Sociedad Civil Intelectual (pp. 79-92). Mendoza: FEPAI.

Rodríguez, M. (2015b). ¿Reforma administrativa o desmembramiento? La reorganización de la Comisión Nacional de Energía Atómica en el marco del Estado Neoliberal en Argentina (1994). Revista Brasileira de História da Ciência, 8(1), 83-99.

Rodríguez, M. (2019). Ciencia y Tecnología para el desarrollo industrial: La consolidación de empresas mixtas en torno a la actividad nucleoeléctrica (1976-2001). En J. Odisio \& M. Rougier (eds.), Estudios sobre planificación y desarrollo económico. Aportes para un diseño institucional estratégico. Buenos Aires: Lenguaje Claro.

Rubio-Varas, M. y Torre, J. D. la. (2014). El Estado y el desarrollo de la energía nuclear en España, c. 1950-1985. En Documentos de Trabajo (DT-AEHE) (N.o 1403). Asociación Española de Historia Económica. Recuperado de https://ideas.repec.org/p/ahe/dtaehe/1403.html

Rubio-Varas, M. y Torre, J. D. la. (2017). Seeking the Perennial Fountain of the World's Prosperity. En The economic history of nuclear energy in Spain (pp. 1-32). Londres: Palgrave.

Sabato, J. (1970). Para el prontuario del Plan Nuclear argentino. Ciencia e Investigación, 1(1), 3245.

Sabato, J. (1973). Quince años de metalurgia en la Comisión Nacional de Energía Atómica. Ciencia Nueva, (15), 1-19.

Sabato, J. (1983). Propuesta de política y organización en ciencia y tecnología. En Encuentro Nacional de Ciencia, Tecnología y Desarrollo (pp. 39-45). Buenos Aires: Centro de Participación política de la UCR.

Sabato, J. (2011). El pensamiento latinoamericano en la problemática ciencia-tecnología-desarrollo-dependencia. Buenos Aires: Ediciones Biblioteca Nacional.

Schneider, B. R. (1999). Las relaciones entre el estado y las empresas y sus consecuencias para el desarrollo: Una revisión de la literatura reciente. Desarrollo Económico, 39(153), 45-77. DOI: $10.2307 / 3467220$

Schorr, M. (2004). Industria y Nación. Poder económico, neoliberalismo y alternativas de reindustrialización en la Argentina contemporánea. Buenos Aires: Edhasa.

Schvarzer, J. (1987). La política económica de Martínez de Hoz. Buenos Aires: Hyspamérica.

Semenov, B. y Oi, N. (1993). Ciclos del combustible nuclear: Ajustes a nuevas realidades. Boletín OIEA, 35(3), 2-7. 
SIGEN. (1994). ENACE S. A. Informe situación empresaria. Buenos Aires: Autor.

Solingen, E. (1993). Macropolitical consensus and lateral autonomy in industrial policy: The nuclear sector in Brazil and Argentina. International Organization, 47(2), 263-298. DOI: 10.1017) S0020818300027946

Subsecretaría de Planificación Energética (1986). Plan energético nacional 1996-2000. Resumen. Buenos Aires: Secretaría de Energía.

Thomas, Hernán, Santos, G. y Fressoli, M. (eds.). (2013). Innovar en Argentina: Seis trayectorias empresariales basadas en estrategias intensivas en conocimiento. Buenos Aires: Lenguaje Claro Editora.

Thomas, S. (1999). El futuro de los mercados de la electricidad ¿Una economía de mercado que realmente funciona o una oligarquía en ciernes? Problemas del Desarrollo. Revista Latinoamericana de Economía, 29(115/116), 105-128.

Thomas, S. (2004). The British Model in Britain: Failing slowly. Londres: University of Greenwich.

Volman, S. (1986). Evaluación de la capacidad industrial argentina y desarrollo de los proveedores para instalaciones nucleares. Argentina Nuclear, 1(5), 11-13.

Zunino, P. (1982, diciembre 17). Para el plan nuclear, 1982 fue difícil pero fructífero. Diario El Tiempo.

Archivos y repositorios consultados

Fondo Documental de la Empresa Nuclear Argentina de Centrales Eléctricas S.A. Archivo Intermedio de la Nación Argentina.

Boletín Oficial de la República Argentina.

Hemeroteca del Departamento de Prensa de CNEA. 